\title{
A Review on Molecular Mechanisms of Wheat (Triticum aestivum L.) and Rice (Oryza sativa L.) against Abiotic Stresses with Special Reference to Drought and Heat
}

\author{
Shraddha Tomar ${ }^{1}$, Manepalli Seetha Babu ${ }^{1}$, Dinkar J. Gaikwad ${ }^{2 *}$ and Sagar Maitra ${ }^{3}$ \\ ${ }^{1}$ Department of Plant Breeding and Genetics, Jawaharlal Nehru Krishi Vishwa Vidyalaya, Jabalpur, Madhya Pradesh, India \\ ${ }^{2}$ Department of Biochemistry and Plant Physiology, Centurion University of Technology and Management, Odisha, India \\ ${ }^{3}$ Department of Agronomy and Agroforestry, Centurion University of Technology and Management, Odisha, India
} "Corresponding author: dinkargaikwad02@gmail.com (ORCID ID: 0000-0002-7930-1628)

Paper No. 902

Received: 22-04-2021

Revised: 28-05-2021

Accepted: 16-06-2021

\begin{abstract}
Plants face to different changes in environmental conditions that keep on challenging its normal growth and acclimatization. Cereal crops play a key role in supplying food and energy to growing population and these crops are bare to substantial environmental changes causing inferior growth and performance. To overcome the abiotic stresses experienced by plants, several differential molecular and physiological responses were evolved that make the plants to mitigate climatic aberrations. Latest technological developments in genetic engineering as well as molecular breeding register a significant role in developing crop varieties and hybrids those are tolerant to environmental aberrations. Several Transgenes and their Quantitative Trait Locus (QTL) were invented and incorporated into crops for superior performances against abiotic factors in cereals like wheat and rice.

\section{HIGHLIGHTS}

(0 Growth and productivity of cereals are hampered due to abiotic stresses and under the present context of climate change there is the need for adoption of suitable measures.

- Understanding molecular mechanisms are important for development of suitable varieties/ hybrids of cereals combatting ill effects of abiotic stresses.
\end{abstract}

Keywords: Wheat, Rice, Abiotic stress, Drought stress, Heat stress

Agricultural production and productivity are severely affecting due to the rapidly changing climate across the globe. Due to growing human population, increasing demand of food is the major challenge of the present and future agriculture (Maitra et al. 2018). For human consumption as well as livestock feed, cereals are the vital source throughout the world for food material and nutritional components. Abiotic stresses due to water, salinity, cold and high temperatures represent the main constraint for agriculture and if affecting during the reproductive stage, cause significant loss in terms of productivity and quality. Abiotic stress is defined as the damaging effect on living organisms by non-living factors in a specific environment. Abiotic stresses usually cause abnormal functioning of protein. The strategy is to obtain superior performance of crop species under abiotic stress conditions by identifying and modifying the molecular mechanisms that take place under environmental extremities. Drought and high-temperature stresses are predominant challenges to sustainable agriculture. Drought

\footnotetext{
How to cite this article: Tomar, S., Babu, M.S., Gaikwad, J.D., and Maitra, S. 2021. A Review on Molecular Mechanisms of Wheat (Triticum aestivum L.) and Rice (Oryza sativa L.) against Abiotic Stresses with Special Reference to Drought and Heat. IJAEB, 14(2): 215-222.

Source of Support: None; Conflict of Interest: None $\infty$
} 
tolerance is the lenience of crops under the shortage of moisture in the cytoplasm when the water content constitutes $\sim 23 \%$ or $\sim 0.3 \mathrm{~g}$ of the fresh and dry tissue, respectively (Sahebi et al. 2018). Drought tolerance mechanisms, including physiological adaptations, morphological acclimation, and cellular adjustments, are regulated by genetic factors (Hossain et al. 2021). During the monsoon period, Eastern India faces shortage in precipitation that adversely impacts on crop production. Efficacious establishment, survival and yield of a crop mainly rely on the adaptation of the crop to abiotic conditions in which the crop grows. Further, the farmers' realization as well as national productions and food security are negatively influenced by the abiotic stresses. According to Kreps et al. (2002), up to $50 \%$ of agricultural production is lost due to different abiotic abnormalities, however, about 10 to $20 \%$ of crop damage is caused by biotic factors. By affecting sink potency and source capacity, stress due to high temperature influences the grain production and quality of wheat. Considering the above, more widespread approaches that comprise quantitative and qualitative studies of gene expression products are essential at the transcriptome, proteome, and metabolome stages. The specific proteins are developed underdistinct abiotic stresses and therefore, precise importance will be given for stresses due to high temperature and soil moisture shortage by proteomics approaches. For enhancing stress tolerance QTL identification plays an important role (Fig. 1).

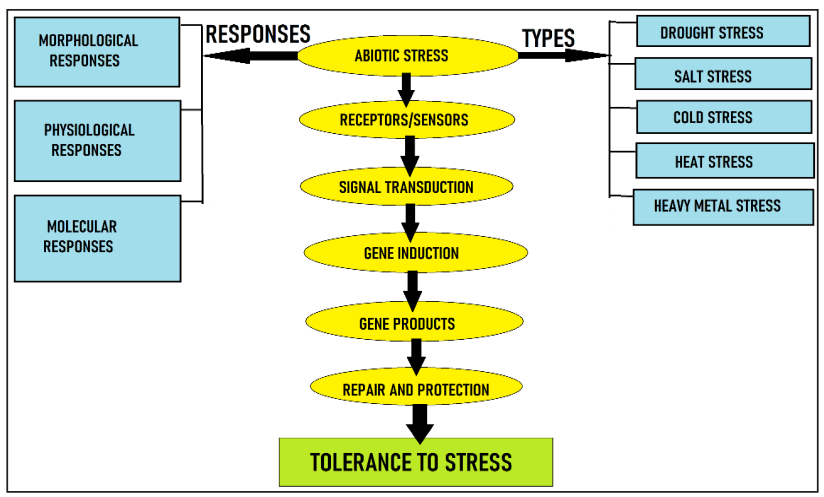

Fig. 1: Abiotic stresses and mechanism of plant molecular responses to environmental stresses for tolerance

To understand the process of plant growth in stress conditions, different functional genomic technologies make it feasible, for instance, metabolomics, proteomics, transcriptomics, and next-generation sequencing. It is feasible to attain an exhaustive and comprehensive analysis by gathering all this information on the mechanism of plant protection against abiotic stresses. There are many genes control drought and these are complex traits, however, the most of these genes make a minor genetic impacts, but for genetically improved plants tolerant to drought these genes are of greater importantance. According to Nezhadahmadi et al. (2013), by the year 2025, close to $65 \%$ of the world's population will live in a drought situation with 1.8 billion people facing complete water shortage.

\section{Response of Wheat to Drought}

Universally, drought is a major problem affecting quality and grain yield in wheat. Drought is caused due to shortage and uneven distribution of rain, soil moisture shortage and higher evapo-transpiration (ET) than soil moisture absorption by the plants. Water deficit signals in the roots during drought and convey the message to the leaves through multiple signalling pathways. The important signalling is caused by abscisic acid (ABA). Drought undesirably influenced the root traits and cell size of the piliferous layer (Adda et al. 2005) and severe drought stress significantly reduced root traits.

\section{Proline}

Proline accumulation occurs in wheat during drought stress and can be formed from pyrroline5-carboxylate synthetase or P5CR (Gowda et al. 2011). According to Shao et al. (2008), there is a close relationship of proline content with soil moisture deficit threshold and wheat anti-drought. In transgenic wheat, stress-induced synthesis of proline helps to develop lenience mechanism to water deficit. According to Vendruscolo et al. (2007), the lenience to water shortage noted was mainly due to defence mechanisms against oxidative stress and not caused due to osmotic adjustment, more precisely in transgenic plants. Proline degradation is catalysed in the mitochondria by proline dehydrogenase (PDH) then $\mathrm{P} 5 \mathrm{C}$ dehydrogenase $(\mathrm{P} 5 \mathrm{CDH})$ by sequential reactions, both of which are induced by gathering of proline in the cells. Thus, genes related to synthesis and degradation of proline are up-regulated where proline is accumulated.

According to Saddique et al. (2020) the speed of rice germplasm screening against water stress can be 
expanded by utilizing the osmotic pressure initiated change in proline substances, Total Antioxidant Capacity (TAC) and the expression of OsP5CS in the rice seedlings as indicator attribute of drought tolerance. Synchronized heat and drought stresses are considered as more hazardous as compared to independently applied heat or water stress. In such cases, external application of proline $(30 \mathrm{mM})$ stimulates the performance of the enzymes like SOD, POD, CAT, TSP and glycine betaine which helps in improvement of photosynthetic pigments and ultimately increases yield of the rice (Hanif, 2021).

\section{Abiotic Stress Gene}

According to Zhang et al. (2007), based on the functioning of methyltransferase and the SAMbinding motif, to transduce stress signals the SAMbinding motif of gene W89 was supposed to be related with other proteins or transcription factors and in an early stage of drought, it finally controls the expression of stress-responsive genes.

\section{Heat Stress}

In wheat, heat stress affects growth and productivity, predominantly at the grain development stage. Schapendonk et al. (2007) showed that the effects of the pre- and post-treatment growing temperature were more than the impact of a 3-day heat shock on yield. HSPs produced to deal with the hightemperature stress in different organisms are known to provide tolerance and repairing of the cellular damage caused by high temperature. Additional carbon assimilates and nitrogen inputs are needed for the inclusion of terminal heat tolerance into high-yielding cultivars (Mitra and Bhatia, 2008).

\section{Molecular Responses of Drought Tolerance in Wheat}

Some genes are manipulated by drought and produced different proteins related to drought stress and enzymes including dehydrins, late embryo abundant (LEA) protein, vacuole acid invertase, and glutathione S-transferase (GST) (Anderson and Davis, 2004), expression of ABA genes and synthesis of proteins like RAB, rubisco, helicase, proline (Fig. 2). Expression of ABA genes and carbohydrate are the molecular basis of drought tolerance. In stressful environments, plants respond by modifying their gene expressions and protein productions. The available details on droughtresponsive genes are still limited as their functions have not been completely determined. The junction stage is susceptible to drought because this phase is the linkage point in the vegetative and flowering growth phase and it is significant for growth and reproduction (Shi et al. 2010).

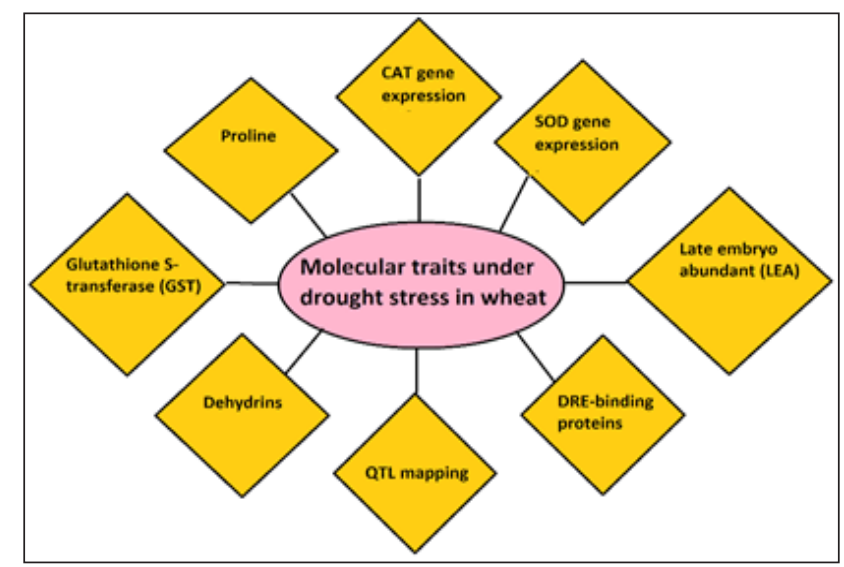

Fig. 2: Molecular traits under drought stress in wheat

According to Sivamani et al. (2000), the HVA1 gene promotes the enhancement of wheat growth under water stress conditions. A protein is formed by the HVA1 gene that is in group 3 LEA and has 11 amino acids. Proline is an essential protein that has the vital function in drought stress tolerance. Hong-Bo et al. (2006) narrated that the proline plays a vital role against drought under water stress conditions. In drought stress, gathering of late embryogenesis abundant (LEA) proteins are inclined and these proteins have been recognized through the amino acid sequence and help other proteins to recover after denaturation during drought stress. Protein constituent of groups one, two, and three of LEA were noticed in wheat. V-PPase genes in wheat, TaVP1, TaVP3, and TaVP2, were identified by Wang et al. (2009). Kam et al. (2007) also noticed the genes which are responsible in wheat for drought stress. In wheat, the Em gene encoded LEA protein the first group has also been reported. Ali-Benaliet al. (2005) reported that group two (dehydrins) proteins and group four of LEA proteins in durum wheat. Transcripts of the Td27e, Td29b, and Td16 genes were saved late in embryogenesis and throughout the development of the seed. In embryo and endosperm, TaRZF74 and TaRZF59 were articulated and TaRZF38 and TaRZF70 were uttered in the 
wheat root at the utmost level. A sequence of putative DREB labelled DREB3A (TaDREB3A, Gene bank ID: AY781349) from wheat to seclude a DREB from wild wheat and for recognition of its function in higher drought resistance (Lucas et al. 2011).

\section{Molecular Responses for Drought Tolerance in Rice}

Rice being the most important staple crop of the world (Ashkani et al. 2015) is grown under various levels of input conditions and provides up to $80 \%$ calories daily to individuals (Khush, 2005). Serraj et al. (2011) reported that nearly 23 million hectares of rain-fed rice face drought stress. By developing drought-tolerant varieties, in the future, it is possible to counteract drought stress in rice. Various abiotic stresses such as drought, salinity, high and cold temperature, etc. affect its yield depending on the environmental situations. Apparently, due to its petite root system, swift stomatal closure and thin cuticular wax rice is considered one of the most drought-susceptible plants. Genes responsible for abiotic tolerance in rice have been revealed that causes genetic variation in it by the characterization and screening of rice germplasm at a different molecular and genetic level under abiotic stress.

For drought tolerance, a wide range of genes are responsible that can be identified through significant advances in model plant species. In this regard, the genome sequencing has been done in various model crops inclusive of major plant species (Feuillet et al. 2011). A number of studies were carried out in this regard such as genome annotation, functional genomics and molecular physiology to find out candidate genes for drought tolerance in several models and major crops. Drought associated candidate genes express various proteins that perform magnificiant roles in: (I) cellular protection by osmotic adjustment, structural adaptation, repair, degradation, and detoxification and (II) positive interactions with other proteins and transcription factors, namely, protein kinases and Bzip, MYB, and DREB associated in drought responses by modifying other responsive genes involved in protection of cell to fight with water stress in plants. Candidate genes should be established via approaches, such as qRT-PCR, expression analysis, linkage mapping, incorporation into QTL maps, TILLING, and allele mining, and applications of these approaches have been reviewed previously (Varshney et al. 2005). One common approach for isolating the candidate genes responsible for drought stress in drought-resistant genotypes is the generation of expressed sequence tags from cDNA libraries of tissues collected under water stress. Another approach to identifying candidate genes is transcriptional profiling that includes the various gene expression analysis in plant tissues after the onset of drought stress, as well as between drought-tolerant genotypes and drought-susceptible genotypes (Hampton et al. 2010).

\section{Management Options}

To survive against abiotic stresses, numerous cultural practices has been long practiced. However, the utilization of the genetic/hereditary tools for this reason is latest inclusion. Previously Plant breeders gave major focus on development of high yielding genotypes which performed better under non stressful condition but at present due to fluctuating climatic conditions plants are more susceptible to different abiotic stresses. In recent studies, researchers have given more focus on developing stress tolerance varieties by practicing conventional and molecular breeding approaches (Farooq et al. 2009).

\section{Conventional Approach}

The choice of appropriate breeding programme for the development of tolerant cultivars to a distinct environmental stress depends on variety of factors such as screening techniques, sources and tolerance mechanisms, approaches of geneaction and heritability, and their association to agronomical traits. Conventional breeding techniques are used to develop drought tolerance lines in many cereals and leguminous crops like wheat, barley, maize, safflower, chickpea, common beans and soybean (Meena et al. 2017). One of the major challenges to apply this methodology is recognizing phenotypes that relate well with abiotic stress tolerance. Considerable work has been done in wheat to produce stress tolerance genotypes. A diploid wild species of bread wheat (Aegilops tauschii) was crossed with a tetraploid (Triticum turgidum) at CIMMYT (Valkoun, 2001). IRRI, Philippines is also working on developing drought tolerant rice cultivars using this typical old technique in plant 
breeding. Similar research has been going on at ICARDA and ICRISAT to introduce the drought tolerant genotypes of major cereal crops (Ashraf, 2010).

\section{Modern Breeding Approach}

Conventional breeding approaches has been effectively utilized to develop abiotic stress tolerance varieties with high production but this practice has many limitations to develop multiple stress tolerant varieties. Marker assisted breeding has opened up opportunity to transfer genetic material from one species to another to identify abiotic stress tolerance traits. This reproducing technique used both subatomic and ordinary ways to deal with consolidate various characteristics followed by rigid choice during every age. This advanced breeding strategy uses both traditional and molecular approaches to integrate multiple traits and strict selection during respective generation. Marker assisted selection has played a very crucial role in development of rice genotypes with two or more genes/QTLs in short time (Pradhan et al. 2015). Dixit et al. (2020) developed seven introgression lines (ILs) using MAS breeding method and claimed that these ILs are performing better than their respective recurrent parents in terms of quality and overall agronomic performances. Dixit et al. (2017) developed drought tolerance NILs of a rice variety sabitri using markerassisted backcross breeding in combination with phenotypic selection. Reynolds et al. (2012) studied several physiological trait based variations to fight against drought and heat stress at different growth phases of the wheat. Sinha et al. (2018) reported that expression analysis of the underlying candidate gene AK248593.1 in meta-QTL26 region discovered two times higher expression in the NILs carrying the co-localized SSR markers and claimed that the linked markers of the several meta-QTL regions linked with different traits can be used for effective transfer of the QTLs through marker assisted selection in wheat breeding.

\section{Transgenic Approach}

To fulfill the increasing need for food and to distinguish the detrimental impacts of abiotic stress on plants, it is crucial to produce transgenic lines that have further developed protection from a wide range of abiotic stresses (Noman et al. 2017). Ali and
Kim (2020) while studing on transgenic breeding approaches for improving abiotic stress predicted that application of CRISPR/Cas9 technology in various plant species could reform second green revolution in agriculture, which could be possible solution to fulfil the increasing demand of food and food security for the growing populations. Since last decade, a bunch of abiotic stress-related genes has effectively been relocated into rice to work on its obstruction against abiotic stresses. Numerous transgenic rice plants with improved abiotic stress-resilience have been identified (Zhao and Zhang, 2007). Huang et al. (2005) claimed that two transgenic varieties namely GM Xianyou 63 and GM-II-Youming 86 required minimal amount of pesticides $(20 \%)$ and shown $6 \%$ increase in the production when compared with wild type rice. Introduction of such transgenic plants diminish the danger of wellbeing risks brought by pesticides, are harmless to the ecosystem and assist with expanding the overall gain for the farmer. In case of transgenic wheat, several genes have been recognized which deliberate drought tolerance and help to survival and plant growth development. Notwithstanding, less examination has been led for the improvement of transgenic wheat when contrasted with other cereal crops which might be due to the complex hereditary attributes of wheat (Araus et al. 2019).

\section{Molecular Breeding for Drought Tolerance}

In cereals, the genetic upgradation against drought greatly hinges onsome factors such as the genetic resources availability, the complexity of the marked production system, selection strategies and the ability for implementing the cost-effective breeding process. Currently, the important focus is on detection and the genetic mapping of major QTLs performing under drought stress, and thus, development of drought resistant genotype marker assisted breeding programs will provide accurate phenotyping and integration of marker (Kumar et al. 2009). The selection of single secondary trait is not desired to be effective for improvement towards drought tolerance (Zhao et al. 2008). Before using marker-assisted selection the identified QTLs for grain yield, morphophysiological traits should carefully confirm. According to Chen et al. (2011), QTL detection with minimum inputs and could also create valuable materials for breeding with wide genetic variability for tolerating drought. 
Table 1: Abiotic stress-responsive proteins in wheat and rice cultivars

\begin{tabular}{lllll}
\hline S1. No. & Species & Functions & Accession no. & Gene Name \\
1 & Triticum aestivum & Heat shock protein 16.9 H & Q42417_WHEAT & hsp16.9- 17LC3 \\
2 & Triticum turgidum subsp. durum & Alpha-tubulin H & gil82174009 & atu3 \\
3 & Triticum aestivum & Aquaporin D,S,C & gil161897630 & PIP1-8 \\
4 & Triticum monococcum & Beta-amylase 1 H & gil148529650 & BAMY1 \\
5 & Oryza sativa subsp. Japonica & Calcineurin B-like protein 10 H,S & CNBLA_ORYSJ & CBL10 \\
6 & Triticum aestivum & Catalase I H & CATA2_WHEAT & CATA \\
7 & Triticum aestivum & Cyclin dependent protein kinase D & gil86438780 & cdc2-1A \\
8 & Oryza sativa subsp. Indica & Cyclin-B2-2 D & CCB22_ORYSI & CYCB2-2 \\
9 & Triticum aestivum & Cyclophilin D,C & gi82547214 & CYP23-d \\
10 & Oryza sativa & Drought-induced S-likeribonuclease D & Q69JX7_ORYSA & P0569E \\
11 & Oryza sativa subsp. japonica & Heat stress transcription factor C-1b H & HFC1B_ORYSJ & HSFC1 B \\
12 & Triticum turgidum subsp. durum & Lipid transfer protein precursor D & Q9FEK9_TRITU & ltp7.1 \\
13 & Triticum aestivum & Small heat shock protein, chloroplastic H & HS21C_WHEAT & HSP21 \\
\hline
\end{tabular}

D: Drought stress; H: Heat stress; S: Salt Stress; C: Cold Stress (Sources: Abu et al. 2010).

The molecular instruments in drought-tolerant plants are inclusive of (i) transcriptional activation and regulation leading to the activation of signal transduction cascade, (ii) proteins such as LEA proteins and chaperones HSPs (heat shock proteins), (iii) biosynthesis of proline, betaine, glycine, mannitol and trehalose, (iv) accelerated synthesis of chemical antioxidants, such as glutathione and ABA, (v) reactive oxygen species (ROS) toxicity reduced by glutathione $S$-transferase and superoxide dismutase (Aprile et al. 2009).

\section{CONCLUSION}

With the increasing population, demand for food and other commodities are also increasing significantly, however, the productivity of these crops is in risk with ever enhancing abiotic stresses that negatively impacting on the food security. Under such challenging circumstances, the selection of the crop varieties tolerant to these types of stresses is considered as achievable alternative to maintain the productivity of the crops in many regions. The role of molecular mechanisms is also widely discussed for each abiotic stress factor. Researchers have focused on mechanism of tolerance to abiotic stresses. Many innovative techniques have been developed to screen and identify the genotype for the tolerance to abiotic factors. Genotype with developed tolerance mechanism to different abiotic stresses may help to produce sustainable yield under stress affected conditions. The cumulative effect of various abiotic stresses has become more complex, and that require new strategies to fight the stress factors for increased crop production. New advanced techniques in sequencing, marker development and genomic analysis have provided the opportunity to review the method of generating populations suitable for analysis.

\section{REFERENCES}

Abu, H.K., Ki-Hyun K., Kwang, H. S., Jong, S. C., Byung, K.B., Hisashi, T., Hwa et al. 2010. Abiotic stress responsive proteins of wheat grain determined using proteomics technique. Aust. J. Crop Sci., 4(3): 196-208.

Adda, A., Sahnoune, M. Kaid-Harch, Mand, Merah, O. 2005. Impact of water deficit intensity on durum wheat seminal roots. Comptes. Rendus. Biol., 328(10-11): 9

Ali, A. and Ju-Kon Kim, 2020. Transgenic Breeding Approaches for Improving Abiotic Stress Tolerance: Recent Progress and Future Perspectives. Int. J. Mol. Sci., 21: 2695.

Ali-Benali, A.M., Alary, R., Joudrier, P. and Gautier, F.M. 2005. Comparative expression of five LEA genes during wheat seed development and in response to abiotic stresses by real-time quantitative RT-PCR. Biochimica et Biophysica Acta, 1730(1): 56-65.

Anderson, V.J. and Davis, G.D. 2004. Abiotic stress alters transcript profiles and activity of glutathione S-transferase, glutathione peroxidase, and glutathione reductase in Euphorbia esula. Plant Physiol., 120(3): 421-433.

Aprile, A., Mastrangelo, M. A. and De Leonardis, M.A. et al. 2009. Transcriptional profiling in response to terminal drought 19 stress reveals differential responses along the wheat genome. BMC Genomics, 10, article no. 279. 
Araus, J.J., Serret, M.D. and Lopes, M.S. 2019. Transgenic solutions to increase yield and stability in wheat: Shining hope or flash in the pan? J. Exp. Bot., 70:1419-1424.

Ashkani, S., Rafi, Y.M. and Shabanimofrad et al. 2015. Molecular breeding strategy and challenges towards improvement of blast disease resistance in rice crop. Front Plant Sci., 6: 886.

Ashraf, M. 2010. Inducing drought tolerance in plants: recent advances. Biotechnol Adv., 28(1): 169-183.

Batool, N., Noor, T., Ilyas, N. and Shahzad, A. 2015. Molecular basis of salt stress tolerance in crop plants. Bolan. Soc. Pure and Appl. Biol., 4: 80-88.

Bhatia, C.R.R.M. 2007. Bioenergetic cost of heat tolerance in wheat crop. Curr. Sci., 94(8): 25.

Chen, M., Ali, J., Fu, B., Xu, J. and Zhao, M. 2011. Detection of drought-related loci in rice at reproductive stage using selected introgressed lines. Agr. Sci. China, 10(1): 1-8.

Dixit, S., Singh, U.M., Singh, A.K. et al. 2020. Marker Assisted Forward Breeding to Combine Multiple Biotic-Abiotic Stress Resistance/Tolerance in Rice. Rice(NY), 13(1): 29.

Dixit, S., Yadaw, R.B., Mishra, K.K. et al. 2017. Marker-assisted breeding to develop the drought-tolerant version of Sabitri, a popular variety from Nepal. Euphytica., 213: 184.

Farooq, M., Wahid, A., Kobayashi, N., Fujita, D. and Basra, S.M.A. 2009. Plant drought stress: effects, mechanisms and management. Agron. Sustain. Dev., 29: 185-212.

Feuillet, C., Leach, E. J., Rogers, J., Schnable, S.P. and Eversole, K. 2011. Crop genome sequencing: Lessons and rationales. Trends Plant Sci., 16(2): 77-88.

Foley, A.J., Ramankutty, N., Brauman, A.K. et al. 2011. Solutions for a cultivated planet. Nature, 478(7369): 337-342.

Fukai, S.M. and Wade, L.J. 1999. How can breeding contribute to more productive and sustainable rainfed lowland rice systems. Field Crop Res., 64(1-2): 199-209.

Gosala, S.S., Wania, S. and Kanga, S.M. 2009. Biotechnology and drought tolerance. J. Crop Improv., 23(1): 19-54.

Gowda, R.P.V., Henry, A., Yamauchi, A., Shashidhar, H.E. and Serraj, R. 2011. Root biology and genetics improvement for drought avoidance in rice. Field Crop Res., 122(1): 1-3.

Gubis, J., Vankova, R., Cervena, V., Dragunova, M., Hudcovicova, M. and Lichtnerova, H. 2007. Transformed tobacco plants with increased tolerance to drought. South Afr. J. Bot., 73: 505-511.

Guo, G.F., Ge, P., Ma, C.Y., Li, X.H., Lv, D.W., Wang, S.L. et al. 2012. Com- parative proteomic analysis of salt response proteins in seedling roots of two wheat varieties. Proteomics J., 75: 1867-1885.

Hampton, M., Xu, W.W. and Kram, W.B. 2010. Identification of differential gene expression in Brassica rapanectaries through expressed sequence tag analysis. PLoS One, 5(1): e8782.

Hanif, S., Saleem, M.F., Sarwar, M. et al. 2021. Biochemically Triggered Heat and Drought Stress Tolerance in Rice by Proline Application. J Plant Growth Regul., 40: 305-312.
Hong-Bo, S., Xiao-Yan, C. and Li, Y. 2006. Investigation on the relationship of proline with wheat anti-drought under soil water deficits. Colloids and Surfaces B, 53(1): 113-119.

Hossain, A., Skalicky, M., Brestic, M., Maitra, S., Alam, M.A., Syed, M.A., Hossain, J., Sarkar, S., Saha, S., Bhadra, P., Shankar, T., Bhatt, R., Chaki, A.K., Sabagh, A.E.L. and Islam, T. 2021. Consequences and mitigation strategies of abiotic stresses in wheat (Triticum aestivum L.) under the changing climate. Agronomy, 11(2): 241.

Huang, J., Hu, R., Rozelle, S. and Pray, C. 2005. Insect-resistant GM rice infarmers' fields: assessing productivity and health effects in China. Science, 308: 688-690.

Kam, J.P., Gresshoff, P., Shorter, S. and Xue, P.G. 2007. Expression analysis of RING zinc finger genes from Triticum aestivum and identification of TaRZF70 that contains four RING-H2 domains and differentially responds to water deficit between leaf and root. Plant Sci., 173(6): 650-659.

Khan, N. and Naqvi, N.F. 2011. Effect of water stress in bread wheat hexaploids. Curr. Res. J. Biol. Sci., 3(5): 487-498.

Khush, S.G. 2005. What it will take to feed 5.0 billion rice consumers in 2030. Plant Mol. Biol., 59(1): 1-6.

Kramer, P.J. and Boyer, S.J. 1995. Water Relations of Plants and Soils. Academic Press, New York, NY, USA.

Kreps, J.A., Wu, Y., Chang, H.S., Zhu, T., Wang, X. and Harper, J.F. 2002. Transcriptome changes for Arabidopsis in response to salt, osmotic, and cold stress. Plant Physiol., 130: 2129-41.

Kumar, A.R.S., McNally, K.L., Slamet-Loedin, I., Bruskiewich, R., Mauleon, R., Cairns, J. and Hijmans, R.J. 2009. Improvement of drought resistance in rice. Adv. Agron., 103: 41-99.

Lucas, S., Durmaz, E., Akpnar, A.B. and Budak, H. 2011. The drought response displayed by a DRE-binding protein from Triticum dicoccoides. Plant Physiol. Biochem., 49(3): 346-351.

Noman, A., Aqeel, M., Deng, J., Khalid, N., Sanaullah, T. and Shuilin, H. 2017. Biotechnological advancements for improving floral attributes in ornamental plants. Front. Plant Sci., 8: 530.

Pradhan, S.K., Nayak, D.K., Mohanty, S., Behera, L., Barik, S.R., Pandit, E., Lenka, S. and Anandan, A. 2015. Pyramiding of three bacterial blight resistance genes for broad-spectrum resistance in deep-water rice variety, Jalmagna. Rice (N Y), 8(1): 51.

Sahebi, M.M., Hanafi, M.M., Rafii, M.Y., Mahmud, T.M.M., Parisa, A. Mohamad, O. et al. 2018. Improvement of Drought Tolerance in Rice (Oryza sativa L.): Genetics, Genomic Tools, and the WRKY Gene Family. BioMed. Res. Int., 2018:3158474. doi:10.1155/2018/3158474.

Sinha, N., Priyanka, V., Ramya, K.T. et al. 2018. Assessment of Marker-trait Associations for Drought and Heat Tolerance in Bread Wheat. Cereal Res. Communi, 46: 639-649.

Maiti. K.R. and Satya, P. 2014. Research advances in major cereal crops for adaptation to abiotic stresses. GM Crops Food, 5(4): 259-279. 
Maitra, S., Zaman, A., Mandal, T.K. and Palai, J.B. 2018. Green manures in agriculture: A review. J. Pharmacogn. Phytochem., 7(5): 1319-1327.

Manschadi, M.A., Christopher, J., Devoil, P. and Hammer, L.G. 2006. The role of root architectural traits in adaptation of wheat to water-limited environments. Funct. Plant Biol., 33(9): 823-837.

Meena, H.P., Bainsla, N.K. and Yadav, D.K. 2017. Breeding for Abiotic Stress Tolerance in Crop Plants, Recent Advances in Plant Stress Physiology, pp. 329-378.

Mitra, R. and Bhatia, C.R. 2008. Bioenergetic cost of heat tolerance in wheat crop. Curr. Sci., 94(8): 25.

Nezhadahmadi, A., Prodhan, H.Z. and Faruq, G. 2013. Drought Tolerance in Wheat. Sci. World J., Article ID 610721, Doi: 10.1155/2013/610721.

Saddique, M.A.B., Ali, Z., Ali Sher, M., Farid, B., Ikram, R.M. and Saeed, A.M. 2020. Proline, Total Antioxidant Capacity, and OsP5CS Gene Activity in Radical and Plumule of Rice are Efficient Drought Tolerance Indicator Traits., Int. J. Agr., Article ID 8862792, 9 pages, 2020. https://doi. org/10.1155/2020/8862792.

Sankar, B., Abdul Jaleel, C., Manivannan, P., Kishorekumar, A., Somasundaram, R. and Panneerselvam, R. 2008. Relative efficacy of water use in five varieties of Abelmoschus esculentus(L.) Moench under water-limited conditions. Colloid Surfaces B., 62(1): 125-129.

Schapendonk, A.H.C.M., Xu, H.Y., VanerPutten, P.E.L. and Spiertz, J.H.J. 2007. Heat-shock effects on photosynthesis and sink-source dynamics in wheat (Triticum aestivum $L$ ). NJAS - Wageningen J. Life Sci., 55(1): 37-54.

Serraj, R., McNally, L.K. and Slamet-Loedin, I. 2011. Drought resistance improvement in rice: An integrated genetic and resource management strategy. Plant Prod. Sci., 14(1): 1-14.

Shao, B., Chud, L.Y., Jaleel, C.A., and Zhao, C.X. 2008. Waterdeficit stress-induced anatomical changes in higher plants. C R Biol., 331(3): 215-25.

Shi, F.J., Mao, G.X., Jing, L.R., Pang, X.B., Wang, G.Y. and Chang, P.X. 2010. Gene expression profiles of response to water stress at the jointing stage in wheat. Agric. Sci. China, 9(3): 325-330.

Shimazaki, Y., Ookawa, T. and Hirasawa, T. 2005. The root tip and accelerating region suppress elongation of the decelerating region without any effects on cell turgor in primary roots of maize under water stress. Plant Physiol., 139(1): 458-465.
Swamy, M.P.B. and Kumar, A. 2013. Genomics-based precision breeding approaches to improve drought tolerance in rice. Biotechnol. Adv., 31(8): 1308-1318.

Valkoun, J. 2001. Wheat pre-breeding using wild progenitors in Wheat in a Global Environment, eds Z. Bedö and L. Láng (Dordrecht: Springer), pp. 699-707. doi: 10.1007/97894-017-3674-9-94.

Varshney, K.R., Graner,A. and Sorrells, E.M. 2005. Genomics assisted breeding for crop improvement. Trends Plant Sci., 10(12): 621-630.

Veesar, F.N., Channa, N.A., Rind, J.M and Larik, S.A. 2007. Influence of water stress imposed at different stages on growth and yield attributes in bread wheat genotypes Triticum aestivum L. Wheat Inform. Service, 104: 15-19.

Vendruscolo, E.C.G., Schuste, I., Pileggi, M., Scapimd, C.A., Molinari, H.B.C., Marure, C.J. et al. 2007. Stress-induced synthesis of proline confers tolerance to water deficit in transgenic wheat. J. Plant Physiol., 164(10): 1367-76.

Wang, Y., Xu, H. and Zhang, G. et al. 2009. Expression and responses to dehydration and salinity stresses of V-PPase gene members in wheat. J. Genetics Genomics, 36(12): $711-720$.

Xu, F., Yuan, S. and Lin, H.H. 2011. Response of mitochondrial alternative oxidase (AOX) to light signals. Plant Signal. Behavior, 6(10): 55-58.

Yamada, M., Morishita, H. and Urano, K. et al. 2005. Effects of free proline accumulation in petunias under drought stress. J. Experim. Bot., 56(417): 1975-1981.

Zhang, R., Xu, Z., Li, L., Chen, M. and Ma,Y. 2007. Isolation and expression analysis of a novel abiotic stress-induced gene W89 from wheat. Agric. Sci. China., 6(4): 391-8.

Zhao, X., Xu, J. and Zhao, M. 2008. QTLs affecting morphphysiological traits related to drought tolerance detected in overlapping introgression lines of rice (Oryza sativa L.). Plant Sci., 174(6): 618-25.

Zhao, F.Y. and Zhang, H. 2007. Transgenic Rice Breeding for Abiotic Stress Tolerance-Present and Future. Chin. J. Biotech., 23(1): 1-6.

Zhu, J., Dong, H.C. and Zhu, K.J. 2007. Interplay between cold responsive gene regulation, metabolism and RNA processing during plant cold acclimation. Curr. Opinion Plant Biol., 10(3): 290-295. 